\title{
Increased expression of transcription factor TFAP2 $\alpha$ correlates with chemosensitivity in advanced bladder cancer
}

Iver Nordentoft ${ }^{1 *}$, Lars Dyrskjøt ${ }^{1}$, Julie S Bødker ${ }^{1}$, Peter J Wild ${ }^{2}$, Arndt Hartmann ${ }^{3}$, Simone Bertz ${ }^{3}$, Jan Lehmann ${ }^{4}$, Torben F Ørntoft ${ }^{1}$ and Karin Birkenkamp-Demtroder ${ }^{1}$

\begin{abstract}
Background: The standard treatment for patients with advanced transitional cell carcinoma of the bladder is platin based chemotherapy. Only approximately $50 \%$ of the patients respond to chemotherapy. Therefore, molecular predictive markers for identification of chemotherapy sensitive subgroups of patients are highly needed. We selected the transcription factor TFAP2 $\alpha$ from a previously identified gene expression signature for chemotherapy response.
\end{abstract}

Methods: TFAP2 $\alpha$ expression and localization was assessed by immunohistochemistry using a tissue microarray (TMA) containing 282 bladder cancer tumors from patients with locally advanced ( $\mathrm{pT} 2-\mathrm{T}_{\mathrm{b}}$ and $\mathrm{N}_{1-3}$ ) or metastatic $\left(M_{1}\right)$ disease. All patients had received cisplatin containing chemotherapy. Furthermore, QPCR analysis of three TFAP2 $\alpha$ isoforms was performed on tumor specimens of advanced muscle invasive bladder cancers (T2-4). Using the bladder cell lines T24 and SW780 the relation of TFAP2 $\alpha$ and cisplatin and gemcitabine sensitivity as well as cell proliferation was examined using siRNA directed TFAP2 $\alpha$ knockdown.

Results: TFAP $2 \alpha$ protein expression was analyzed on a TMA with cores from 282 advanced bladder cancer tumors from patients treated with cisplatin based combinational chemotherapy. TFAP2 $\alpha$ was identified as a strong independent predictive marker for a good response and survival after cisplatin-containing chemotherapy in patients with advanced bladder cancer. Strong TFAP2 $\alpha$ nuclear and cytoplasmic staining predicted good response to chemotherapy in patients with lymph node metastasis, whereas weak TFAP2 $\alpha$ nuclear staining predicted good response in patients without lymph node metastasis. In vitro studies showed that siRNA mediated knockdown of TFAP2 $\alpha$ increased the proliferation of SW780 cells and rendered the cells less sensitive to cisplatin and gemcitabine. In contrast to that T24 bladder cells with mutated p53 showed to be more drug sensitive upon TFAP2 $\alpha$ depletion.

Conclusions: High levels of nuclear and cytoplasmic TFAP2 $\alpha$ protein were a predictor of increased overall survival and progression free survival in patients with advanced bladder cancer treated with cisplatin based chemotherapy. TFAP2 $\alpha$ knockdown increased the proliferation of the SW780 bladder cells and reduced cisplatin and gemcitabine induced cell death. The inverse effect was observed in the TP53 mutated T24 cell line where TFAP2 $\alpha$ silencing augmented cisplatin and gemcitabine sensitivity and did not stimulate proliferation.

\footnotetext{
* Correspondence: iver.nordentoft@ki.au.dk

${ }^{1}$ Molecular Diagnostic Laboratory, Department of Molecular Medicine, Aarhus University Hospital, Aarhus, Denmark

Full list of author information is available at the end of the article
} 


\section{Background}

Bladder cancer is the fifth most common malignancy in Europe and the fourth most common malignancy in the United States. The most prevalent histological type is transitional cell carcinoma (TCC), which constitutes up to $95 \%$ of the malignancies of the bladder. About $30 \%$ of TCC's display a solid, invasive growth pattern, being either locally advanced (pT3a, pT3b, pT4 and/or pN1, $\mathrm{pN} 2$, pN3 M0) or metastatic $\left(\mathrm{M}_{1}\right)$ at the time of diagnosis or at later visits [1]. The response rate to chemotherapy is only approximately $50 \%$ [2]. Presently, there are two standard chemotherapeutic regimens: MVAC (methotrexate, vinblastine, doxorubicin, and cisplatin) or GC (gemcitabine and cisplatin). Median survival is 14 to 15 months, and 5-year overall survival rate is between $13 \%$ and $15 \%$ [3]. Although the gemcitabine and cisplatin combination has a significantly better toxicity profile, both regimens still carries risk for significant toxicity and toxic deaths [4] and a substantial fraction of patients will suffer from adverse reactions without achieving any benefit. It is therefore of utmost importance to be able to discriminate between responders and non-responders for improved selection of patients to chemotherapy and to improve the individual patient's quality of life. A molecular signature for chemo-resistance, based on microarray profiling of cancer specimens has been described for locally advanced and/or metastatic bladder cancer [5]. BIRC5 (survivin) and BSG (emmprin) were validated as independent predictive markers for response and survival after cisplatin-containing chemotherapy by immunohistochemistry in an independent material of 124 patients with locally advanced $\left(\mathrm{T}_{4 \mathrm{~b}}\right.$ and $\left.\mathrm{N}_{2-3}\right)$ or metastatic $\left(\mathrm{M}_{1}\right)$ disease [5]. In the present study we investigated another interesting molecule from this gene expression signature, the transcription factor Activator Protein TFAP2 $\alpha$. TFAP2 $\alpha$ belongs to the TFAP2 family of transcription factors that in humans and mice consist of five members, TFAP $2 \alpha$, TFAP $2 \beta$, TFAP $2 \gamma$, TFAP $2 \delta$ and TFAP $2 \varepsilon$. Orthologs show a similarity between 60 and $99 \%$ at the amino-acid level. The TFAP2 family is characterized by having a highly conserved helix-span-helix dimerization motif at the carboxyl terminus together with a central basic region and a less conserved proline/glutamine rich domain at the amino terminus. The helix-span-helix domain facilitates homo and heterodimerization between the TFAP2 members. Once dimerized, the helix-spanhelix motif and the neighboring basic domain facilitate DNA binding and the N-terminal proline/glutamine-rich domain mediates transactivation. The TFAP2 family members participate in the regulation of many signaling pathways and are essential during embryogenesis and development. TFAP2 proteins participate in tumorigenesis through regulation of neoplasia associated genes such as P21, Rb, TP53, ER $\alpha$, BCL2, cKIT, MMP-2, E-cadherin and c-myc (reviewed in [6,7]). TFAP2 $\alpha$ knockout mice are not viable and have severe ventral body wall closure defects (thoracoabdominoschisis)[8]. Currently many studies have linked deregulated TFAP $2 \alpha$ activity to malignant transformation. The TFAP $2 \alpha$ gene locus at 6 p22 is frequently lost in various cancers [9]. Lost or decreased TFAP $2 \alpha$ expression has been identified in human cancers of the breast, colon, prostate, ovary and brain [10-15] suggesting TFAP2 $\alpha$ to be a tumor suppressor gene. Immunohistochemical analysis demonstrated a correlation between decreased TFAP2 $\alpha$ expression and advanced colon adenocarcinomas [11]. In breast cancer, low nuclear TFAP2 $\alpha$ expression is associated with disease progression and elevated metastatic capability [12]. Furthermore, reduced TFAP $2 \alpha$ expression predicted elevated risk of recurrent disease in breast cancer [16]. Moreover, re-expression of TFAP2 $\alpha$ in metastatic melanoma cells decreased their tumorigenity and inhibited their metastatic potential in nude mice [17]. So far the importance of TFAP2 $\alpha$ in transitional cell carcinomas of the bladder has not been described. In the present study, we showed that high levels of nuclear and cytoplasmic TFAP $2 \alpha$ protein were associated with increased overall survival and progression free survival of patients with lymph node positive advanced bladder cancer after cisplatin based chemotherapy. Furthermore, we showed that siRNA mediated knockdown of TFAP2 $\alpha$ stimulated proliferation of the SW780 bladder cell line along with decreased cisplatin and gemcitabine sensitivity.

\section{Methods}

Archival formalin-fixed paraffin embedded (FFPE) tissues of cystectomy specimens were derived from a previously reported Phase III, multicenter randomized control trial of two different adjuvant chemotherapy regimens, $\mathrm{AUO}-\mathrm{AB}$ 05/95 conducted by the Arbeitsgemeinschaft Urologische Onkologien (AUO) collaborative group of the Federal Republic of Germany $[18,19]$. The clinicopathological characteristics of all patients were reviewed by one surgical pathologist (A.H.). Tumor staging was performed according to the criteria of the International Union against Cancer (UICC). Representative haematoxylin- and eosinstained (HE) slides of formalin-fixed and paraffinembedded tissue blocks obtained at cystectomy where reviewed and the tumor area was marked. Tissue microarrays (TMA) where constructed by obtaining a $1.5 \mathrm{~mm}$ punch-biopsy from each tumor. TMA construction was done as described previously [20]. The TMAs were stained with $\mathrm{HE}$ and were reviewed for the presence of representative tumor tissue by one surgical pathologist. The study was approved by the local ethical committee of the University Regensburg. 


\section{Expression Plasmids}

Plasmids of the human TFAP $2 \alpha$ isoform 2 GenBank [NM_001032280] and 3 GenBank [NM_001042425]: pcDNA3.1/V5-His-TFAP2 $\alpha v 2$ and pcDNA3.1/V5-HisTFAP $2 \alpha$ v3 were generated by PCR amplification (Expand High Fidelity PCR System (Roche)) of bladder cancer patient $\mathrm{CDNA}$ as template using the primer pairs: 5'-GCCACCATGTTAGTTCACAGTTTTTCAGCC-3', 5'-CTTTCTGTGCTTCTCCTCTTTGTCACTG-3' and 5' - GAGCCGCGATGTCCATACTTGCC -3', 5'-CTTT CTGTGCTTCTCCTCTTTGTCACTG-3' respectively and cloned into the pcDNA3.1/V5-His ${ }^{\odot} \mathrm{TOPO}^{\odot} \mathrm{TA}$ expression vector (Invitrogen) following manufactures instructions.TFAP $2 \alpha$ isoform 1 GenBank[NM_003220] was generated by sub-cloning from pcDNA3.1/V5-HisTFAP $2 \alpha$ v3 using primer pair 5'-CCACCATGCTTTGGAAATTGACGGATAATATCAAGTACGAGGACTGCGAGGACCGTCAC-3' and 5'-CTTTCTGTGCTTC TCCTCTTTGTCACTG-3'. The PCR product was subsequently cloned as described above.

\section{Western blotting}

Whole cell protein lysates from COS7, T24 and SW780 were collected using a cell scraper and lysed in RIPA buffer (50 mM TRIS pH 7.5, $150 \mathrm{mM} \mathrm{NaCl}, 1 \%$ NP40, and 0.5\% Deoxycholic Acid, sodium salt) supplemented with complete protease inhibitor cocktail (Boehringer Mannheim). Protein concentration was determined using Bradford reagent (BioRad laboratories, HerculesCA, USA). A $25 \mu \mathrm{g}$ sample was resolved by SDS-PAGE $(12 \%$ Tris- $\mathrm{HCl}$, Invitrogen). Proteins were transferred to PVDF membrane (Millipore) and blocked overnight with 3\% w/v skimmed milk powder in PBS buffer supplemented with $0.05 \%$ Tween-20. Membranes were probed with primary antiTFAP2 $\alpha$ mouse IgG (Abcam, ab18112 1:500) and subsequently, secondary horseradish peroxidase-conjugaed goat anti-mouse (Dako Cytomation 1:5000). The immunoreactive bands were visualized using ECL plus (Amersham Biosciences) and UVP ChemiDoc-It, Imaging system (UVP Inc.)

\section{Cell Culture}

Bladder cell lines T24 and SW780 were obtained from American Type Culture Collection (ATCC-LGC standards, Borås, Sweden), were re-authenticated via STR analysis using the Cell-ID-system (G9500, Promega, Nacka, Sweden), products were analysed on an AppliedBiosystems3130 Genetic Analyser. No mycoplasma contamination was detected using nested PCR-based mycoplasma detection. COS-7 cells (green monkey) were cultured in RPMI 1640 and T24 (P53 Y126*, HRAS G12V) and SW780 bladder cells in DMEM with L-glutamine (Gibco, Invitrogen Corporation, Carlsbad, CA, USA) supplemented with $10 \%$ fetal bovine serum
(Gibco), $100 \mathrm{U} / \mathrm{ml}$ penicillin (Gibco) and $100 \mathrm{U} / \mathrm{ml}$ streptomycin (Gibco) and maintained in a humidified atmosphere at $37{ }^{\circ} \mathrm{C}$ and $5 \% \mathrm{CO}_{2}$.

\section{Transient transfection}

The day prior to transfection $5 \times 10^{5}$ COS-7, $6 \times 10^{5}$ T24, $6 \times 10^{5}$ SW780 cells in T25 culture flasks $(5 \mathrm{ml}$ culture medium), $28 \times 10^{3} \mathrm{~T} 24,42 \times 10^{3} \mathrm{SW} 780$ cells in 24 -well plates $\left(500 \mu\right.$ l culture medium), $4 \times 10^{3} \mathrm{~T} 24,6 \times 10^{3}$ SW780 cells in 96 -well (100 $\mu$ l culture medium). Plasmid transfections were performed using FuGENE 6 (Roche) and siRNA transfections were performed using Lipofectamin 2000 both following the manufacturer's instructions. TFAP2 $\alpha$ specific siRNA was obtained from Ambion (ABI; Foster City, CA)(Cat\# s14003). Non-targeting siRNA was obtained from Dharmacon as Non-TARGET \#2 siCONTROL (Cat\# D-001208-14-20).

\section{Isolation of RNA}

T24 and SW780 RNA was isolated from 6-well and 24-well plates using RNeasy spin columns (Qiagen, Hilden, Germany), as recommended by the manufacturer. RNA was quantified by measuring absorbance at 260 and $280 \mathrm{~nm}$.

\section{Cell Proliferation Assay}

Cell proliferation was measured using the CyQUANT cell proliferation assay (Invitrogen) according to the manufacturer's instructions. Fluorescence measurements were made using a microplate reader (Labsystems Multiscan $\mathrm{MCC} / 340$ ) with excitation at $485 \mathrm{~nm}$ and emission detection at $530 \mathrm{~nm}$.

\section{Cell viability assay}

The viability of sub-confluent cells was analyzed by 3-(4,5dimethylthiazole-2-yl)-2,5-diphenyltetrazolium bromide (MTT) reduction assay. The assay was performed in 96 well plates seeding 4000-6000 cell/well in $200 \mu \mathrm{l}$. After $24 \mathrm{~h} 100 \mu \mathrm{l}$ culture medium was carefully removed and $25 \mu \mathrm{l}$ MTT solution was added (1 g MTT (Sigma M5655) dissolved in $200 \mathrm{~mL}$ D-PBS.) and stored shielded from light $1.5 \mathrm{~h}$ at $37^{\circ} \mathrm{C}$ and then $100 \mu \mathrm{l}$ solubilization (50\% dimethylformamide, 20\% SDS) buffer was added and left protected from light ON. Readout was done using a microplate reader (Labsystems Multiscan MCC/340) at $540 \mathrm{~nm}$. Absorbance at $692 \mathrm{~nm}$ was used as reference.

\section{Real-time RT-PCR}

One $\mu \mathrm{g}$ of total RNA was used for cDNA synthesis with random hexamer primers using the SuperScript ${ }^{\circledR}$ II Reverse Transcriptase (Invitrogen Calsbad, CA, USA) according to the manufacturer's instructions. Total RNA at 10-50 ng per $10 \mathrm{uL}$ of reaction mixture was used for measurement of the target mRNA. The real-time RT-PCR assay was performed using the ABI 7500 FAST machine 
(ABI;Foster City, CA). 10 ul real-time RT-PCR reactions consists of 5 ul $2 \times$ TaqMan $^{\circledR}$ FAST Universal Master Mix $(\mathrm{P} / \mathrm{N}$ 43660783, ABI; Foster City, CA), 0.5 ul $20 \times$ TaqMan ${ }^{\circledR}$ Assay/probe (ABI;Foster City, CA)) and cDNA equivalent to 10-50 ng of total RNA in $4.5 \mathrm{ul} \mathrm{H2O}$. Thermal FAST cycle program was: $20 \mathrm{~s}$ at $95^{\circ} \mathrm{C}$ followed by 40 cycles of $3 \mathrm{~s}$ at $95^{\circ} \mathrm{C}$ and $30 \mathrm{~s}$ at $60^{\circ} \mathrm{C}$. Reactions were set up in triplicate for each sample, and gene expressions were normalized to eukaryotic Ubiquitin $C$ using SYBR GREEN PCR Master Mix (Applied Biosystems) and primers 5'-GATTTGGGTCGCGGTTCTT-3', 5'-TGCCTTG ACATTCTCGATGGT-3' [21]. All assays were carried out in 96-well format plates covered with optical adhesive cover $(\mathrm{P} / \mathrm{N} 4346906$ and $\mathrm{P} / \mathrm{N} 4311971 \mathrm{ABI}$, Foster City, CA). We used the $2^{-\Delta \Delta C T}$ method to calculate the relative gene expression. TaqMan ${ }^{\circledR}$ Assays used were: Hs01029410_m1 (TFAP2 $\alpha$ isoform 1,2 and 3); Hs00231461_m1 (TFAP2 $\alpha$ isoform); Hs01033609_m1 (TFAP2 $\alpha$ isoform).

\section{Growth curves via RT-CES system (RTCA)}

The T24 or SW780 cells were harvested and transferred into 16-well DP or 96-well SP E-Plates of the RTCA system, which contain electrodes integrated into the bottom surfaces of each well that measure cell index based on impedance using the RT-CES system (ACEA Biosciences, San Diego, CA, USA. This technology has recently been acquired by Roche Applied Science and is being marketed under the name xcelligence System). Changes in cell status such as cell number, viability and adherence were monitored in real time and quantified by detecting sensor electrical impedance. Cell index correlates with the number of cells attached to the bottom of the plate. For the experiments reported here, the number of cells added to each well varied from 4000 to 6000 per well with a total volume of $200 \mu \mathrm{l}$ of media.

\section{Immunohistochemistry}

Immunohistochemical analysis of FFPE bladder tissue sections $(4 \mu \mathrm{m})$ and tissue microarray sections were performed essentially as described in [22]. The primary antibody against TFAP2 $\alpha$ was ab18112 (mouse monoclonal, dilution 1:40, Abcam). Core biopsies of the TMA were either evaluated according to cytoplasmic staining (weak or strong intensity) or nuclear staining (weak or strong intensity). The TMA's were scored blinded and independently by two observers. In case of disagreement, the core was reevaluated and consensus was reached.

\section{Statistical analysis}

All data calculations were done using the STATA statistical software (version 10).

\section{Results}

Expression of the individual variants of TFAP $2 \alpha$ in advanced bladder cancer specimens

A real-time Q-PCR analysis was performed using cDNA from 10 tumor specimens of advanced muscle invasive bladder cancer (T2-4) to analyze the expression of the TFAP $2 \alpha$ transcript variants. The analysis confirmed that all three variants of TFAP $2 \alpha$ were present in all of the 10 tested bladder tumors (Figure 1A). The largest isoform TFAP $2 \alpha-1$ was most abundantly expressed compared to TFAP $2 \alpha$ isoform 2 and 3. The mRNA level of TFAP $2 \alpha$ isoform 1 was approximately 8 and 16 fold higher than isoform 2 and 3 respectively $(p<0.005)$. There is no statistical difference in expression levels between the mRNA level of isoform 2 and 3 .

\section{TFAP $2 \alpha$ expression in bladder carcinomas}

To assess the biological significance of TFAP $2 \alpha$ expression in bladder tumors, we evaluated the expression
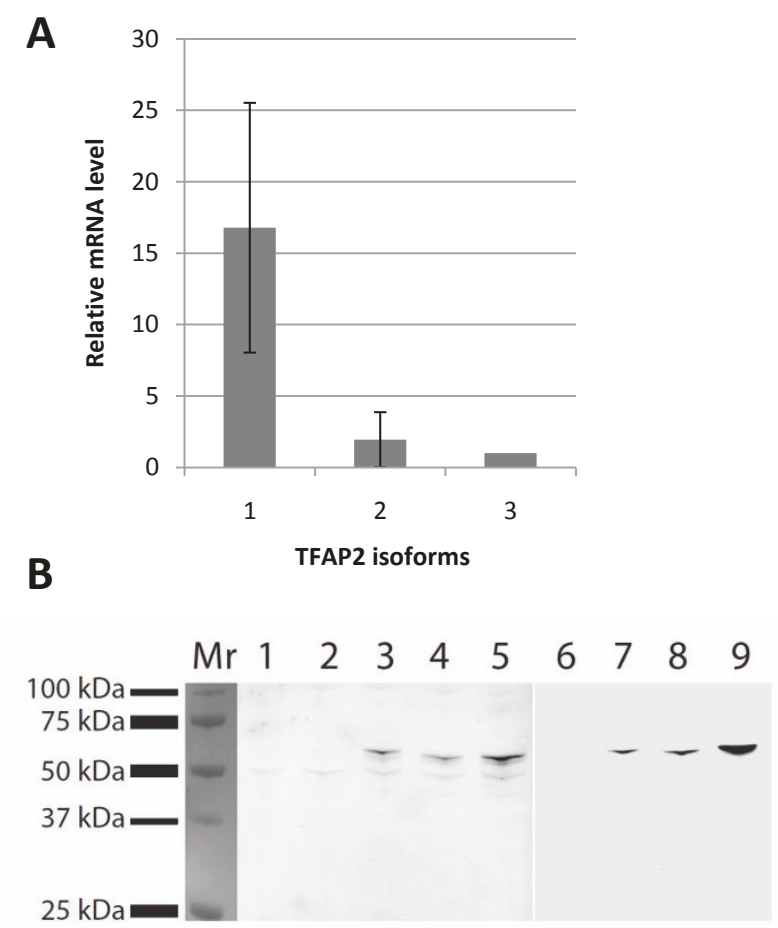

Figure 1 Expression of TFAP $2 \alpha$ isoforms. (A) Expression of TFAP $2 \alpha$ isoform 1, 2 and 3 in advanced muscle invasive bladder cancer (T2-4) were determined using real-time Q-PCR. Analysis was performed on cDNA from 10 tumor specimens and each bar represents the mean from the 10 samples.(B) COS-7 cells were transiently transfected with empty pcDNA3.1N5-His vector (lane 2, 6), pcDNA3.1N5-His-TFAP2 $\alpha$ isoform 1(lane 3, 7), isoform 2 (lane 4, 8) and isoform 3 (lane 5,9 ). Western blot of $30 \mu \mathrm{g}$ total protein lysate from non-transfected HU609 bladder cells (lane 1) and COS-7 transfected cells (lane 2-9) $48 \mathrm{~h}$ post transfection probed with anti TFAP2 $\alpha$ antibody (lane 1-5) or anti-V5 antibody (lane 6-9). 
using a TMA containing tumors from 282 patients with advanced bladder cancer with full clinical annotation (Table 1). The antibody specificity was tested successfully using western blots on total protein cell lysates (Figure 1B). Protein extracts of cell lysates from COS-7 cells transiently transfected with constructs containing one of the three different variants of TFAP $2 \alpha$ isoforms 1-3 were used to evaluate the specificity of the antibodies against TFAP2 $\alpha$. The antibody detects all three isoform (lane 3-5) variants of the protein. The predicted MW of TFAP $2 \alpha$ is $52 \mathrm{KDa}$ in agreement with a slightly denser band detected due to the fusion of the V5 tag of the pcDNA3.1 cloning vector. The endogenous TFAP $2 \alpha$ protein is seen below the fusion protein (lane 1-5). The TMA stained for TFAP2a yielded kappa values of ( $\kappa=$ 0.75 cytoplasmic expression) and ( $\kappa=0.68$ nucleic expression) between two observers, examples of typical staining patterns are shown in Figure 2. Kaplan-Meier survival analysis using all samples on the TMA showed no statistical association between overall survival or progression free survival, and cytoplasmic or nuclear TFAP2 $\alpha$ staining. However, Kaplan-Meier survival analysis restricted to patient samples with lymph node

Table 1 Patient Characteristics

\begin{tabular}{ll}
\hline Tumor category & \\
\hline pTis7pT1 pN+ & $8(2.9)$ \\
pT2 pN0 & $2(0.7)$ \\
pT2 pN+ & 34 \\
& $(12.2)$ \\
pT3 pN0 & 95 \\
pT3 pN+ & $(34.2)$ \\
pT4a pN0 & 89 \\
pT4a pN+ & $(32.0)$ \\
* & $23(8.3)$ \\
Age & $27(9.7)$ \\
Sex & $61.3(28.8-79.2)$ \\
\hline Male & \\
Female & 221 \\
& $(78)$ \\
& $61(22)$
\end{tabular}

\begin{tabular}{ll} 
Nodal Status & \\
\hline $\mathrm{pNO}$ & 124 \\
& $(44)$ \\
$\mathrm{pN}+$ & 158 \\
& $(56)$
\end{tabular}

\begin{tabular}{lll}
$\begin{array}{l}\text { Survival } \\
\text { parameters }\end{array}$ & $\begin{array}{l}\text { Progression-free } \\
\text { survival }\end{array}$ & $\begin{array}{l}\text { Overall } \\
\text { Survival }\end{array}$ \\
\hline $\mathrm{pNO}$ & 32.9 & 34.9 \\
$\mathrm{pN}+$ & 21.5 & 24.5 \\
\hline
\end{tabular}

*The stage was missing on four patients. invasion showed that both strong nuclear and cytoplasmic staining correlated with increased overall survival ( $p$ $=0.026$ and $\mathrm{p}=0.002$ respectively (Figure $3 \mathrm{~A}, \mathrm{~B})$ and progression free survival $(p=0.027$ and $p=0.002$ respectively; Figure $3 \mathrm{C}, \mathrm{D})$. Interestingly, analysis of the samples without lymph node invasion showed an inverse correlation for the nuclear staining. Low nuclear TFAP $2 \alpha$ staining was associated with increased overall survival rate $(\mathrm{p}=0.048$; Figure $3 \mathrm{E})$. The cytoplasmic staining showed no correlation to outcome for the non lymph node invasive group ( $\mathrm{p}=0.57$; Figure $3 \mathrm{~F}$ ).

\section{Cisplatin and Gemcitabine sensitivity of bladder Cancer cell lines}

Studies of breast and colon cell lines show improved drug sensitivity of cells strongly expressing TFAP2 $\alpha[11,12]$. Furthermore, TFAP $2 \alpha$ mediates its growth inhibition both directly through TP53 interaction and independently of TP53. In addition, TFAP $2 \alpha$ binds directly to TP53 and stimulates p21 expression [23]. As our immunohistochemical analysis demonstrated decreased chemo sensitivity of bladder cancer patients with lymph node invasion having low TFAP $2 \alpha$ expression we investigated if TFAP $2 \alpha$ silencing would render bladder cancer cells less prone to chemotherapy drugs. The TP53 wild type bladder cancer cell line SW780 and the TP53 mutated bladder cancer cell line T24 were chosen to investigate the role of TFAP $2 \alpha$ in chemo sensitivity. IC50 values of T24 and SW780 upon Cisplatin and Gemcitabine treatment were determined by an MTT viability assay (Figure $4 \mathrm{ABCD})$. For Cisplatin the IC50(48 h) was around $10 \mu \mathrm{M}$ for T24 and $15 \mu \mathrm{M}$ for SW780. Gemcitabine had an IC50 (48 h) of $15 \mu \mathrm{M}$ for T24 and $50 \mu \mathrm{M}$ for SW780. To verify the drug response we monitored the effect on cell proliferation in real time during drug incubation (Figure 4). Cisplatin was added after $24 \mathrm{~h}$ affecting the cells in a time period between 40-60 hours post-addition. For the T24 cells cisplatin concentration of $4 \mu \mathrm{M}$ or more inhibit growth (the max cell index decreased, Figure 4E). At $72 \mathrm{~h}$ that corresponded to the MTT measurement the cell index of the $10 \mu \mathrm{M}$ growth curve is 2.1 compared to 4.4 for the control curve without cisplatin. This approximately 50\% reduction showed that the decreased surface area of the T24 cell population roughly corresponded to the viability readout. SW780 did not show the same association, but in agreement with the viability assay cisplatin inhibited cell growth substantially from $8 \mu \mathrm{M}$ and $16 \mu \mathrm{M}$ decreased growth heavily within the chosen 72 time window (Figure 4F).

\section{TFAP $2 \alpha$ depletion decreased chemotherapy sensitivity in TP53 WT cells}

T24 and SW780 cells were transfected with siRNA targeting TFAP $2 \alpha$ transcript, yielding a 70\% knock down $48 \mathrm{~h}$ 

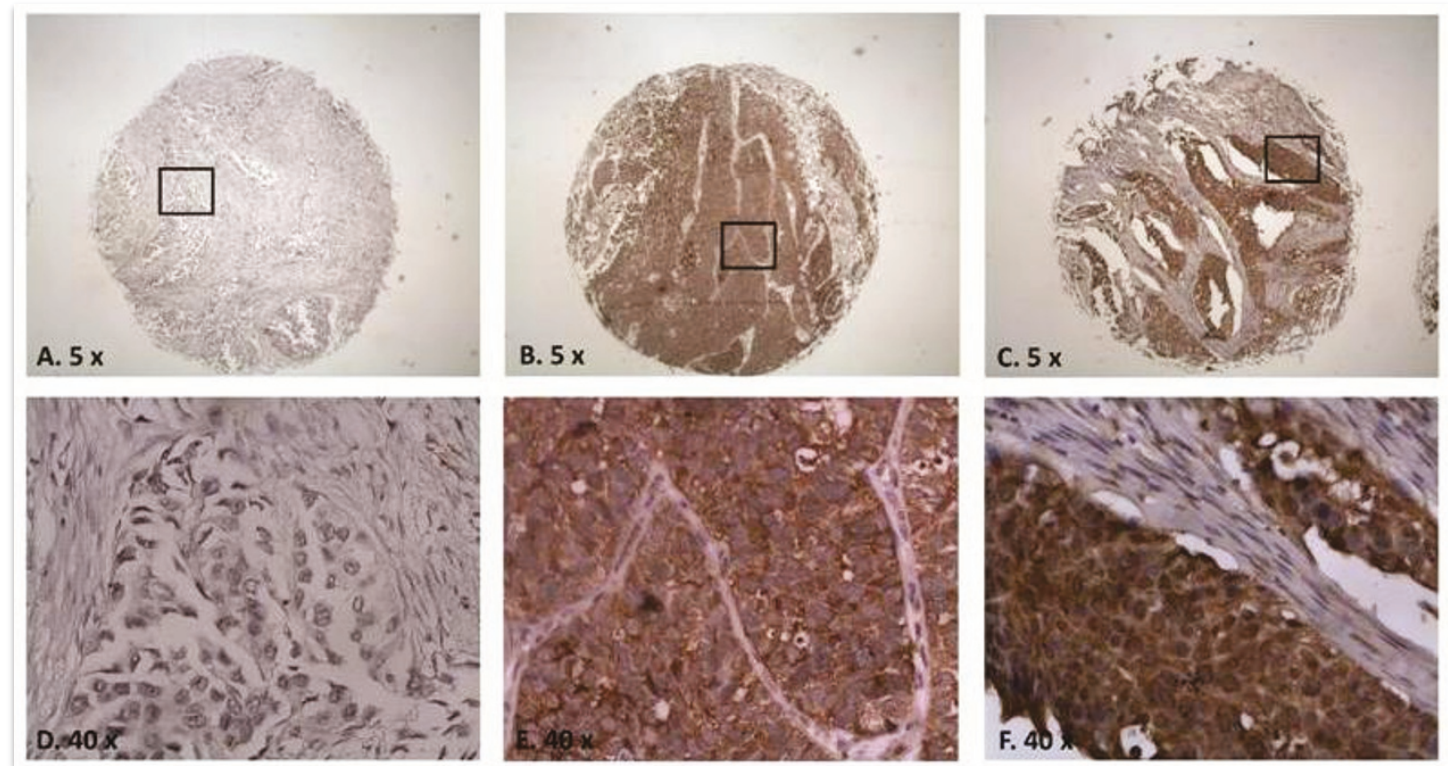

Figure 2 Immunohistochemistry of bladder cancer tissue with antibody against TFAP2 $\alpha$. TFAP2 $\alpha$ immunoreactivity of muscle invasive bladder cancer tumors. A and D: Negative stain of nucleus and cytoplasm at 5 and 40 fold objective magnification, respectively. B and E: Negative stain of nucleus and positive stain of cytoplasm ( $5 \times$ and $40 x$, respectively). C and F: Positive stain of nucleus and cytoplasm (5x and 40x, respectively).

OS analysis lymph node invasive

A

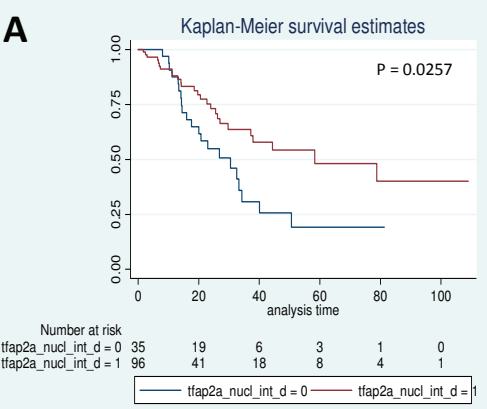

B

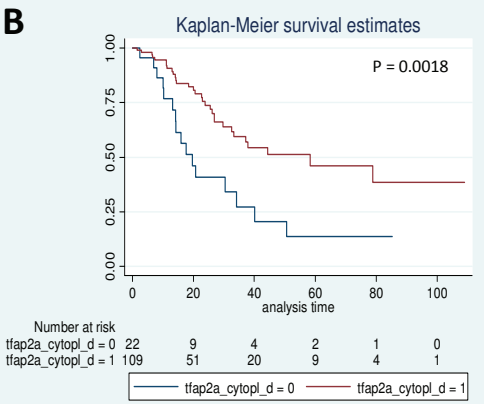

PFS analysis lymph node invasive

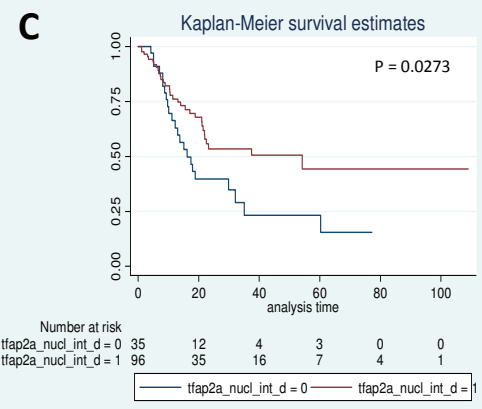

D

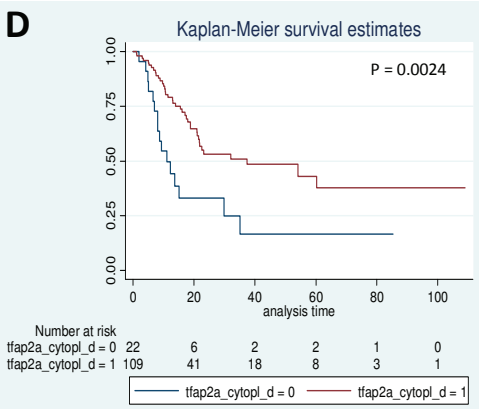

OS analysis no lymph node invasion

$E$
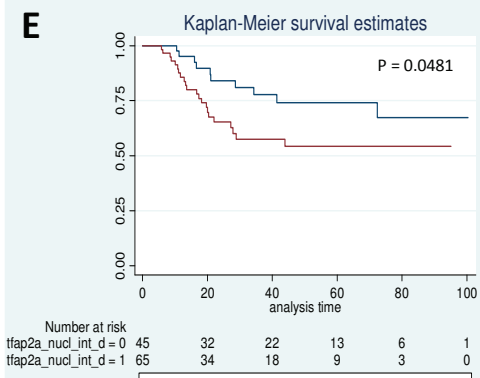

$\mathbf{F}$

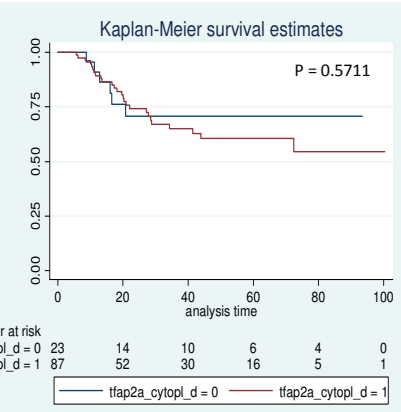

Nuclear staining (ACE); Cytoplasmatic staining (BDF)

Figure 3 The relationship between TFAP $2 \alpha$ staining and survival after chemotherapy. TFAP2 $\alpha$ immunoreactivity and overall survival (OS) rates and progression free survival (PFS). A and B: separation of OS (overall survival) curves based on TFAP2 $\alpha$ nuclear and cytoplasmic staining in the lymph node invasive group, respectively. C and D: separation of PFS curves based on TFAP2 $\alpha$ nuclear and cytoplasmic staining in the lymph node invasive group, respectively. C and F: separation of OS curves based on TFAP2 $\alpha$ nuclear and cytoplasmic staining in the non lymph node invasive group, respectively. Red curves are high TFAP2 $\alpha$ staining and blue curves are low TFAP2 $\alpha$ staining. 


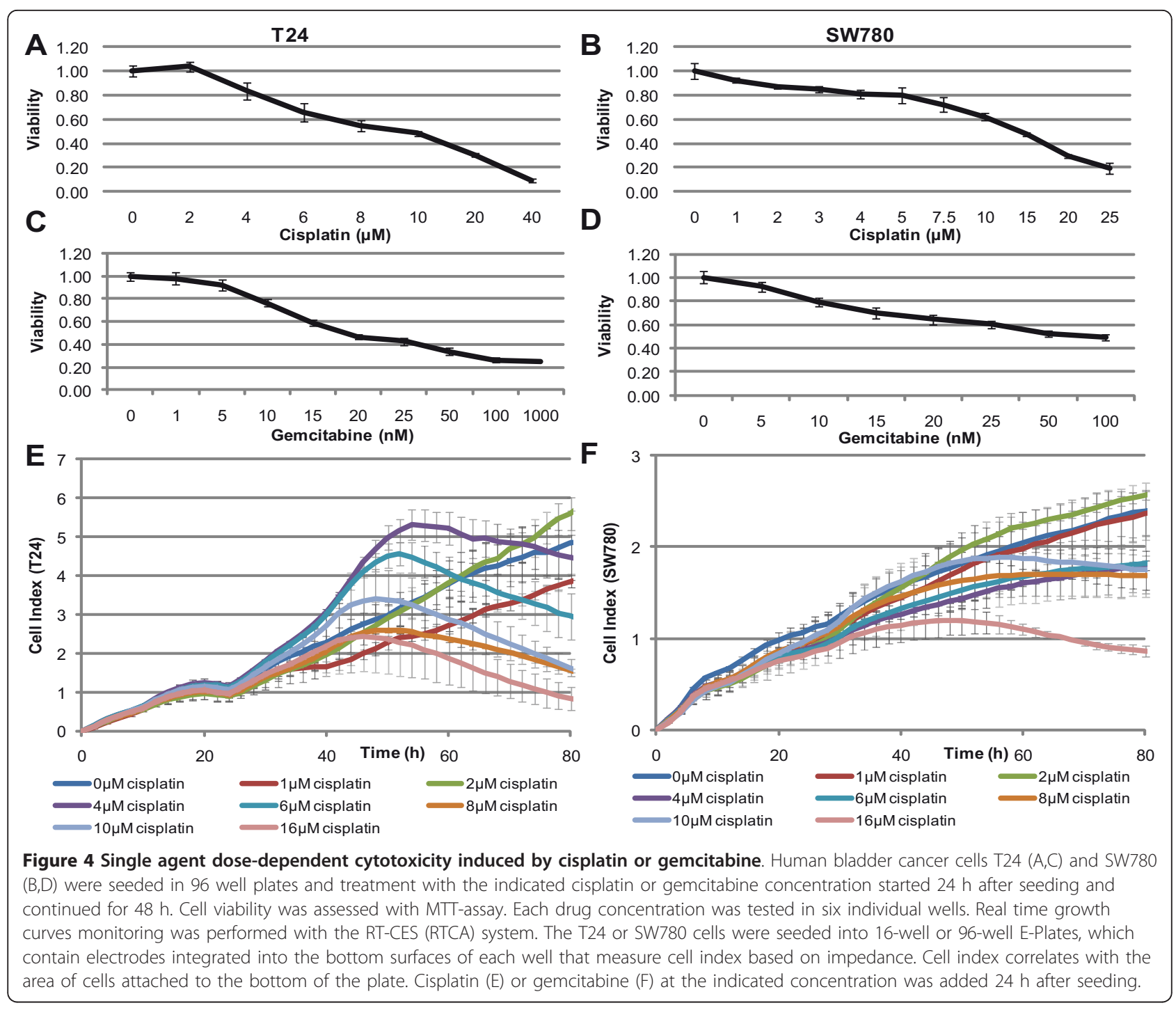

post transfection (Figure $5 \mathrm{AB}$ ). In order to perform the drug incubation without the cells getting too confluent the T24 and SW780 cells were first transfected in T25 culture flask and subsequently after $48 \mathrm{~h}$ incubation the cells were harvested and reseeded in 96 well plates. This extended time period did not have an impact on the knock down (data not shown). Next we added cisplatinor gemcitabine and incubated for $48 \mathrm{~h}$ followed by viability analysis (Figure 5C-F). TFAP2 $\alpha$ silencing rendered SW780 less sensitive against cisplatin and gemcitabine induced cell death (Figure 5D and 5F), however in T24 cells the opposite effect was observed (Figure $5 \mathrm{C}$ and $5 \mathrm{E}$ ).

\section{TFAP $2 \alpha$ affected cell proliferation}

Visual inspection of the TFAP $2 \alpha$ siRNA transfected SW780 cells showed a higher cell population compared to the SW780 cells transfected with siRNA control. To investigate the effect of TFAP $2 \alpha$ siRNA knockdown on proliferation we performed real time proliferation analysis (Figure 6A and 5B). The real time proliferation monitoring of T24 showed no difference between the TFAP $2 \alpha$ siRNA transfected and the siRNA control (Figure 6A) and the addition of cisplatin after $24 \mathrm{~h}$ did not change the proliferation rate either. The TFAP $2 \alpha$ siRNA transfected SW780 cells however were significant proliferating faster than the siRNA control (Figure 6B). Addition of cisplatin after $24 \mathrm{~h}$ decreased the proliferation rate, especially for the TFAP $2 \alpha$ siRNA knockdown. In conclusion TFAP $2 \alpha$ downregulation stimulated proliferation of SW780 cell but not proliferation of T24 cells. Proliferation studies using a CyQuant proliferation assay confirmed these results (Figure 6C and 6D). 


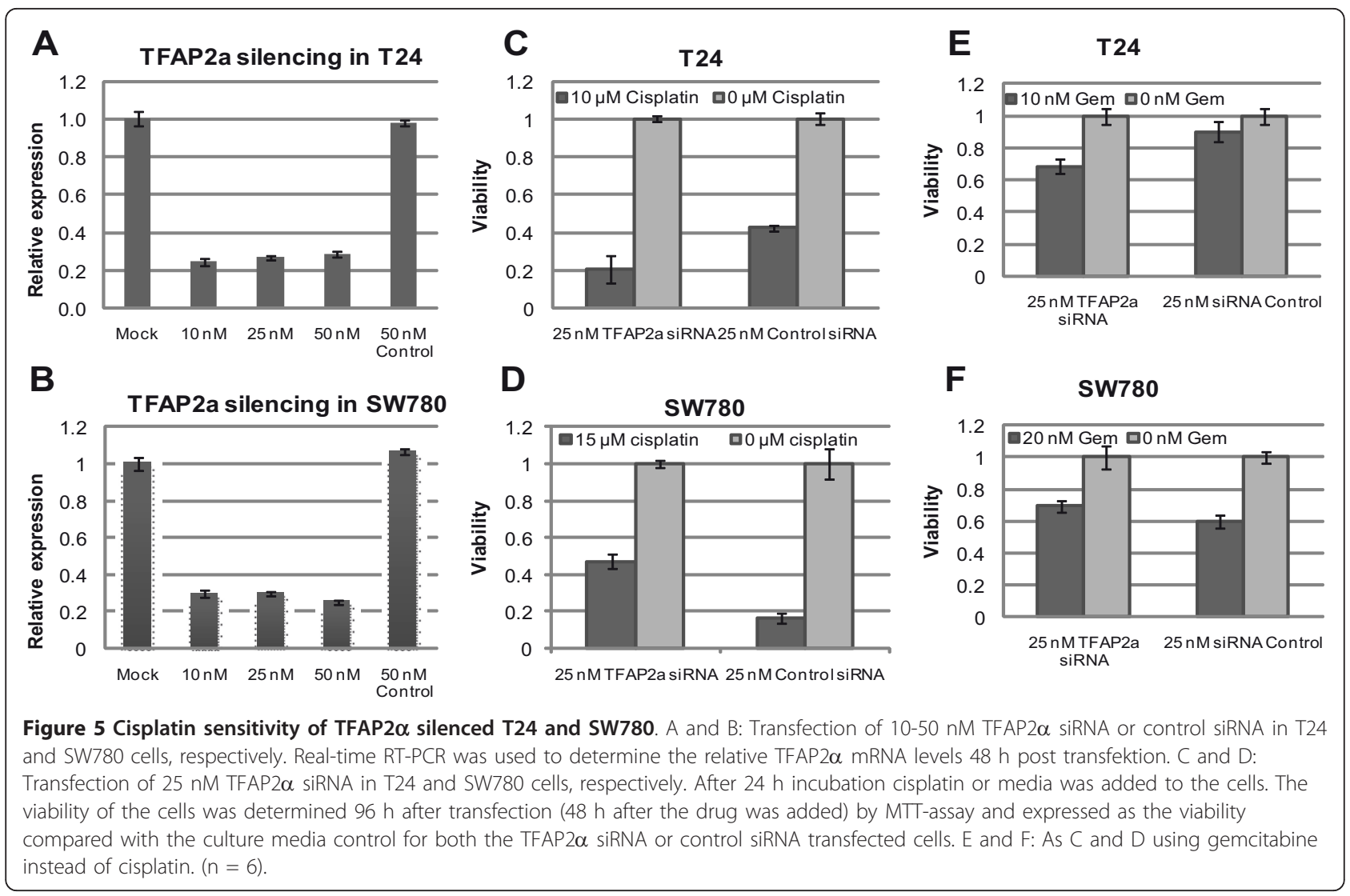

\section{Discussion}

Studies investigating TFAP2 $\alpha$ expression in human cancers show a correlation between reduced nuclear expression of TFAP $2 \alpha$ and shorter recurrence-free survival and aggressive clinicopathological features in colon cancer [11], breast cancer [16], ovarian cancer [13] and melanoma [24]. Several in vitro studies demonstrated that TFAP $2 \alpha$ has an inhibitory effect on cell proliferation and tumorigenesis and proposed that TFAP $2 \alpha$ has a tumor suppressive effect in malignancies, although articles with opposing conclusions also have been published [7]. A large breast cancer study with immunohistochemical detection of TFAP $2 \alpha$ revealed that a reduced level of TFAP $2 \alpha$ in the nucleus and/or a shift of the protein to the cytoplasm may predict a shorter recurrence-free survival and breast cancer-related survival [7]. The observed correlation between high nuclear TFAP $2 \alpha$ staining and decreased overall survival rate for the non lymph node invasive subgroup is in agreement with a breast cancer study from Finland that demonstrated that particular for lymph node positive patients low nuclear TFAP $2 \alpha$ expression is associated with disease progression and elevated metastatic capability [25]. Reduced TFAP $2 \alpha$ expression also predicted elevated risk of recurrent disease in breast cancer
[16]. A study of human melanomas showed that high level of TFAP $2 \alpha$ expression in the cytoplasm relative to the nucleus correlates with poor prognosis. The loss of nuclear TFAP2 $\alpha$ expression was associated with malignant transformation and progression of melanoma, indicating that translocation of TFAP $2 \alpha$ from the cytoplasm to the nucleus may be disrupted during melanoma progression. Thus it seems that the major deficiency in TFAP $2 \alpha$ activity in metastatic melanoma is the loss of nuclear translocation. This could be due to modifications in the nuclear-pore complexes or in the activity of the transport receptors (karyopherines/importins/exportins) or changes of the TFAP $2 \alpha$ protein itself. In our study, both low nuclear and cytoplasmic expression predicted poor outcome, suggesting that decrease of TFAP $2 \alpha$ transcription/ translation or increased turnover rate are a more likely course than translocation in the case of bladder cancer. For the group of patients without lymph node invasion the association was shifted, high nuclear staining was associated with decreased survival time. Although the correlation is not highly significant the difference from the lymph node invasive group is striking. There is no other study analyzing the TFAP $2 \alpha$ staining in an isolated group of patients without lymph node invasion. The results of the 

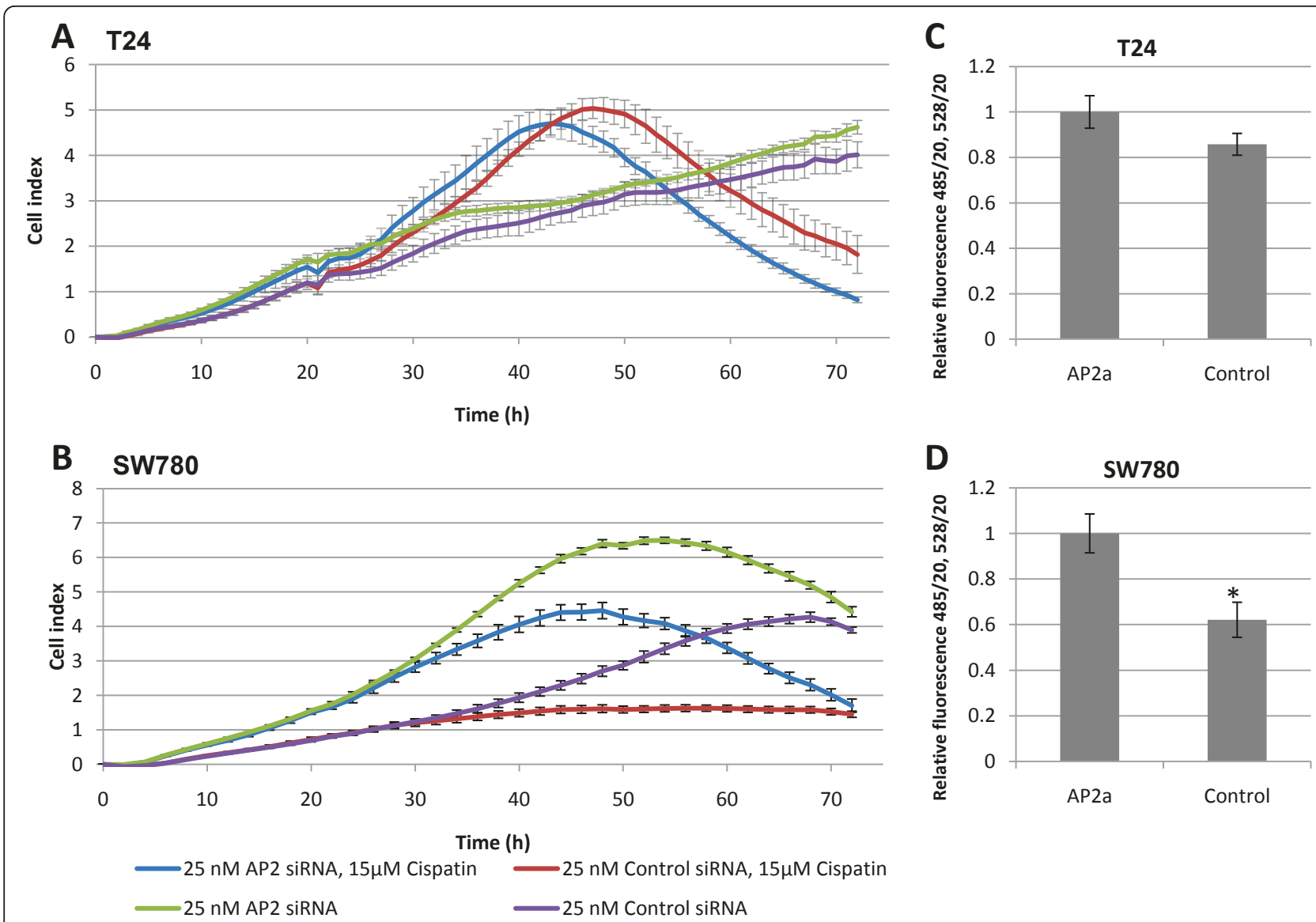

Figure 6 Cell proliferation of TFAP2 $\alpha$ silenced T24 and SW780. Real time growth curves monitoring was performed with the RT-CES system The T24 or SW780 cells were seeded into 16-well or 96-well E-Plates, which contain electrodes integrated into the bottom surfaces of each well that measure cell index based on impedance. Cell index correlates with the area of cells attached to the bottom of the plate. A and B: Transfection of 25 nM TFAP2 $\alpha$ siRNA in T24 and SW780 cells, respectively. $(n=3)$ After $24 \mathrm{~h}$ incubation cisplatin or media was added to the cells. C and D: Transfection of 25 nM TFAP2 $\alpha$ siRNA in T24 and SW780 cells, respectively. After 48 h incubation CyQuant assay was performed. (n $=8)$

TFAP2 $\alpha$ staining analysis raised the question if the decreased chemo sensitivity of bladder cancer patients with lymph node invasion having low TFAP2 $\alpha$ staining is due to depletion of TFAP2 $\alpha$ or deregulation of a downstream target. We performed knock down experiments in bladder cell lines and subsequently measured their sensitivity against cisplatin and gemcitabine. Previous studies have shown that the tumor suppressor activity of TFAP2 $\alpha$ is mediated through a direct interaction with TP53. Furthermore, TFAP2 $\alpha$ induces TP53 dependent $p 21$ transcriptional activation. This supports the observed ability of TFAP2 $\alpha$ to induce G1 and G2 cell cycle arrest in TP5 $3^{+/+}$ but not in TP53 $3^{-/-}$HCT116 colon cells [23]. In contrast to this, a study with breast cancer cell lines demonstrated that TFAP2 $\alpha$ down regulation decreases chemosensitivity irrespective of their TP53 status [26]. In light of these observations, we decided to use a TP53 mutated and a TP53 wild type bladder cell line to conduct functional chemosensitivity studies. We chose the T24 (TP53 homozygous Y126*) and the SW780 TP53 wild type line, both having approximately the same dose response profile of cisplatin and gemcitabine making them appropriate to compare. We showed that TFAP2 $\alpha$ silencing rendered SW780 less sensitive against cisplatin and gemcitabine induced cell death and potentiated the cell death of T24 cells. Moreover, we found that TFAP2 $\alpha$ down regulation stimulated proliferation of SW780 cell and did not change the proliferation rate of T24 cells. The SW780 cell line metastasizes to regional lymph nodes in nude mice tumor transplants, corresponding to the clinical findings in lymph node positive patients $[27,28]$. In contrast, TFAP $2 \alpha$ silencing augments cisplatin and gemcitabine sensitivity and did not stimulate proliferation in the TP53 mutated and non-tumorigenic T24 bladder cell line, corresponding to clinical findings in lymph node negative patients. As mammalian cell terminal differentiate they undergo cell cycle arrest exiting from the cell cycle. TFAP $2 \alpha$ mediates its role as a differentiation associated transcription factor 
through positive regulation of p21 thereby negatively regulating the cell cycle. TFAP $2 \alpha$ induces expression of $\mathrm{p} 21$. The $\mathrm{p} 21$ promoter contains a TFAP $2 \alpha$ binding site located at -103 and -95 where TFAP $2 \alpha$ binds directly and stimulate expression [29]. In addition TFAP $2 \alpha$ targets the p21 promoter in the p53 binding region at -2250 , however only in the presence of p53 in agreement with TFAP $2 \alpha$ has been shown to bind P53 in vivo and in vitro $[23,30]$. Furthermore TFAP2 $\alpha$ induces p21 dependent P53 expression corroborating the observed ability of TFAP $2 \alpha$ to induce G1 and G2 cell cycle arrest [23,31]. The explanation for the aberrant chemosensitivity of TFAP $2 \alpha$ silenced T24 and SW780 could therefore be due to deregulation of the p53/p21 pathway in T24. For SW780, TFAP2 $\alpha$ knockdown may suppress $\mathrm{p} 53 / \mathrm{p} 21$ activation because TFAP $2 \alpha$ is a positive regulate of $\mathrm{p} 53 / \mathrm{p} 21$ expression. This potentiates the effect of cisplatin and gemcitabine as well as relief part of the suppression mediated by p53/p21 on the cell cycle. Taken together, our findings in T24 and SW780 cells may suggest that TFAP $2 \alpha$ down regulation in bladder cells decreased cisplatin and gemcitabine sensitivity in a p53/p21 dependent manner. This is in line with studies showing that knock down of the TFAP $2 \alpha$ expression in breast cancer and colon cancer cell lines resulted in significant reduction in chemotherapy-induced apoptosis $[26,32]$. In non-small cell lung cancer, expression of p53 and p21(Waf1) in mediastinal lymph node specimens were significantly related to the response to platinum chemotherapy [33]. Moreover, overexpression of TFAP $2 \alpha$ expression in a breast cancer cell line augmented increased chemosensitivity and induced endogenous TFAP2 $\alpha$ protein levels in a posttranscriptional way [26]. Within our study, re-introduction of TFAP $2 \alpha$ in T24 and SW780 was performed by transient and stable transfection, Transient transfection was very low $(<20 \%)$ as monitored by QPCR/WB and the selected clones seem to loose TFAP $2 \alpha$ as no increased in transcript was measured (QPCR) compared to the mock transfected. In the literature, re-introduction of TFAP $2 \alpha$ into TFAP $2 \alpha$-negative SW480 colon cancer cells stimulates expression of E-cadherin and down regulation of the MMP-9 expression and leads to dramatic loss of cellular invasive potential in vitro. Interestingly, node positive colorectal cancers showed significant losses for p21 and E-cadherin compared to node negative cancer [34]. TFAP2 $\alpha$ directly binds to the promoter of E-cadherin, where it has been previously reported to act as a transcriptional activator [35]. High E-cadherin expression has been reported to increase cisplatin and gemcitabine sensitivity in pancreatic cancer [36]. Furthermore stable transfectants expressing TFAP $2 \alpha$ in the SW480 cell line significantly inhibited their growth in an orthotopic animal model [35]. Previous studies have also demonstrated that re-expression of TFAP $2 \alpha$ in SW480 cells resulted in an inhibition of colony formation in vitro and upregulation of $\mathrm{p} 21^{\text {Waf1/Cip1 }}[31]$.

\section{Conclusions}

In this study we demonstrated that high levels of nuclear and cytoplasmic TFAP $2 \alpha$ protein was a predictor of improved overall survival and progression free survival of patients with locally advanced bladder cancer undergoing cisplatin based chemotherapy treatment when focusing on the lymph node invasive subgroup. In contrast, high nuclear TFAP $2 \alpha$ staining was associated with decreased overall survival rate for the patients without lymph node metastases. Moreover, siRNA directed knock down of TFAP2 $\alpha$ stimulated proliferation of SW780 bladder cells and decreased their cisplatin and gemcitabine sensitivity. On the contrary, TFAP $2 \alpha$ silencing potentiated cisplatin and gemcitabine sensitivity and did not stimulate proliferation in the TP53 mutated T24 bladder cell line. Future studies are needed to further validate the predictive potential of TFAP $2 \alpha$ expression in bladder cancer. Currently, we are collecting bladder tumor samples (locally advanced T3-4, N1-3 and/or metastatic M1) from patients that have been treated with cisplatin based chemotherapy and which have been characterized according to the RECIST response criteria. This cohort will be used to evaluate if TFAP $2 \alpha$ staining and expression are predictive for cisplatin response in addition to survival.

\section{Acknowledgements}

We would like to acknowledge Michael Stöckle Department of Urology, Saarland University, Homburg/Saar, Germany for involvement in the clinical trial. We thank Pamela Celis, Susanne Bruun, GitteHøj, Lisbet Kjeldsen and Rudolf Jung for excellent technical assistance.

Grant support: The John and Birthe Meyer Foundation; Fritz, Georg, and Marie Cecilie Glud's Foundation; The Oticon Foundation; The University of Aarhus; The Danish cancer foundation

\section{Author details}

'Molecular Diagnostic Laboratory, Department of Molecular Medicine, Aarhus University Hospital, Aarhus, Denmark. ${ }^{2}$ Institute of Pathology, University Hospital Zürich, Zürich, Switzerland. ${ }^{3}$ Department of Pathology, University of Erlangen, Erlangen, Germany. ${ }^{4}$ Urology Practice Prüner Gang, Kiel, Germany.

\section{Authors' contributions}

IN designed experiments, performed experiments, interpreted results, drafted manuscript. JSB designed experiments, performed experiments. LDA conducted statistical analysis, critical revision to manuscript. PJW, AH, SB provided TMA, critical revision to manuscript. JL conducted the clinical trial and provided the clinical data, critical revision of the manuscript. TF $\varnothing$ designed experiments, interpreted results and critical revision to manuscript. KBD designed experiments, optimized IHC, analyzed TMAs, interpreted results, and critical revision to manuscript

All authors have read and approved the final manuscript.

\section{Competing interests}

The authors declare that they have no competing interests.

Received: 25 October 2010 Accepted: 14 April 2011

Published: 14 April 2011 


\section{References}

1. Kaufman DS, Shipley WU, Feldman AS: Bladder cancer. Lancet 2009 374:239-249.

2. Vaishampayan U: Systemic therapy of advanced urothelial cancer. Curr Treat Options Oncol 2009, 10:256-266.

3. Von Der MH, Sengelov L, Roberts JT, Ricci S, Dogliotti L, Oliver T, et al: Long-term survival results of a randomized trial comparing gemcitabine plus cisplatin, with methotrexate, vinblastine, doxorubicin, plus cisplatin in patients with bladder cancer. J Clin Oncol 2005, 23:4602-4608.

4. Von Der MH, Hansen SW, Roberts JT, Dogliotti L, Oliver T, Moore MJ, et al: Gemcitabine and cisplatin versus methotrexate, vinblastine, doxorubicin, and cisplatin in advanced or metastatic bladder cancer: results of a large, randomized, multinational, multicenter, phase III study. J Clin Oncol 2000, 18:3068-3077.

5. Als AB, Dyrskjot L, Von Der MH, Koed K, Mansilla F, Toldbod HE, et al: Emmprin and survivin predict response and survival following cisplatincontaining chemotherapy in patients with advanced bladder cancer. Clin Cancer Res 2007, 13:4407-4414.

6. Eckert D, Buhl S, Weber $S$, Jager $R$, Schorle $H$ : The AP-2 family of transcription factors. Genome Biol 2005, 6:246.

7. Pellikainen JM, Kosma VM: Activator protein-2 in carcinogenesis with a special reference to breast cancer-a mini review. Int J Cancer 2007, 120:2061-2067.

8. Brewer S, Williams T: Loss of AP-2alpha impacts multiple aspects of ventral body wall development and closure. Dev Biol 2004, 267:399-417.

9. Piao Z, Lee KS, Kim H, Perucho M, Malkhosyan S: Identification of novel deletion regions on chromosome arms $2 q$ and $6 p$ in breast carcinomas by amplotype analysis. Genes Chromosomes Cancer 2001, 30:113-122.

10. Berger AJ, Davis DW, Tellez C, Prieto VG, Gershenwald JE, Johnson MM, et al: Automated quantitative analysis of activator protein-2alpha subcellular expression in melanoma tissue microarrays correlates with survival prediction. Cancer Res 2005, 65:11185-11192.

11. Ropponen KM, Kellokoski JK, Pirinen RT, Moisio KI, Eskelinen MJ, Alhava EM, et al: Expression of transcription factor AP-2 in colorectal adenomas and adenocarcinomas; comparison of immunohistochemistry and in situ hybridisation. J Clin Pathol 2001, 54:533-538.

12. Pellikainen J, Naukkarinen A, Ropponen K, Rummukainen J, Kataja V, Kellokoski J, et al: Expression of HER2 and its association with AP-2 in breast cancer. Eur J Cancer 2004, 40:1485-1495.

13. Anttila MA, Kellokoski JK, Moisio Kl, Mitchell PJ, Saarikoski S, Syrjanen K, et al: Expression of transcription factor AP-2alpha predicts survival in epithelial ovarian cancer. Br J Cancer 2000, 82:1974-1983.

14. Heimberger AB, McGary EC, Suki D, Ruiz M, Wang H, Fuller GN, et al: Loss of the AP-2alpha transcription factor is associated with the grade of human gliomas. Clin Cancer Res 2005, 11:267-272.

15. Lipponen P, Aaltomaa S, Kellokoski J, Ala-Opas M, Kosma V: Expression of activator protein 2 in prostate cancer is related to tumor differentiation and cell proliferation. Eur Urol 2000, 37:573-578

16. Gee JM, Robertson JF, Ellis IO, Nicholson RI, Hurst HC: Immunohistochemical analysis reveals a tumour suppressor-like role for the transcription factor AP-2 in invasive breast cancer. J Pathol 1999, 189:514-520

17. Bar-Eli M: Gene regulation in melanoma progression by the AP-2 transcription factor. Pigment Cell Res 2001, 14:78-85.

18. Lehmann J, Retz M, Wiemers C, Beck J, Thuroff J, Weining C, et al: Adjuvant cisplatin plus methotrexate versus methotrexate, vinblastine, epirubicin, and cisplatin in locally advanced bladder cancer: results of a randomized, multicenter, phase III trial (AUO-AB 05/95). J Clin Oncol 2005, 23:4963-4974

19. Hoffmann AC, Wild P, Leicht C, Bertz S, Danenberg KD, Danenberg PV, et al: MDR1 and ERCC1 expression predict outcome of patients with locally advanced bladder cancer receiving adjuvant chemotherapy. Neoplasia 2010, 12:628-636.

20. Lottner C, Schwarz S, Diermeier S, Hartmann A, Knuechel R, Hofstaedter F, et al: Simultaneous detection of HER2/neu gene amplification and protein overexpression in paraffin-embedded breast cancer. J Pathol 2005, 205:577-584

21. Andersen $\mathrm{CL}$, Jensen $\mathrm{L}$, Orntoft TF: Normalization of real-time quantitative reverse transcription-PCR data: a model-based variance estimation approach to identify genes suited for normalization, applied to bladder and colon cancer data sets. Cancer Res 2004, 64:5245-5250.
22. Birkenkamp-Demtroder K, Wagner L, Brandt SF, Bording AL, Gartner W Scherubl $H$, et al: Secretagogin is a novel marker for neuroendocrine differentiation. Neuroendocrinology 2005, 82:121-138.

23. McPherson LA, Loktev AV, Weigel RJ: Tumor suppressor activity of AP2alpha mediated through a direct interaction with p53. J Biol Chem 2002, 277:45028-45033

24. Karjalainen JM, Kellokoski JK, Eskelinen MJ, Alhava EM, Kosma VM: Downregulation of transcription factor AP-2 predicts poor survival in stage I cutaneous malignant melanoma. J Clin Oncol 1998, 16:3584-3591.

25. Pellikainen J, Naukkarinen A, Ropponen K, Rummukainen J, Kataja V, Kellokoski J, et al: Expression of HER2 and its association with AP-2 in breast cancer. Eur J Cancer 2004, 40:1485-1495.

26. Wajapeyee N, Raut CG, Somasundaram K: Activator protein 2alpha status determines the chemosensitivity of cancer cells: implications in cancer chemotherapy. Cancer Res 2005, 65:8628-8634

27. Kyriazis AP, Kyriazis AA, McCombs WB III, Kereiakes JA: Biological behavior of human malignant tumors grown in the nude mouse. Cancer Res 1981, 41:3995-4000.

28. Kyriazis AA, Kyriazis AP, McCombs WB III, Peterson WD Jr: Morphological, biological, and biochemical characteristics of human bladder transitional cell carcinomas grown in tissue culture and in nude mice. Cancer Res 1984, 44:3997-4005.

29. Zeng $Y X$, Somasundaram K, el-Deiry WS: AP2 inhibits cancer cell growth and activates p21WAF1/CIP1 expression. Nat Genet 1997, 15:78-82.

30. Modugno M, Tagliabue E, Ardini E, Berno V, Galmozzi E, De BM, et al: p53dependent downregulation of metastasis-associated laminin receptor. Oncogene 2002, 21:7478-7487.

31. Wajapeyee N, Somasundaram K: Cell cycle arrest and apoptosis induction by activator protein 2alpha (AP-2alpha) and the role of p53 and p21WAF1/CIP1 in AP-2alpha-mediated growth inhibition. J Biol Chem 2003, 278:52093-52101

32. Orso F, Penna E, Cimino D, Astanina E, Maione F, Valdembri D, et al: AP2alpha and AP-2gamma regulate tumor progression via specific genetic programs. FASEB J 2008, 22:2702-2714.

33. Mohamed S, Yasufuku K, Nakajima T, Hiroshima K, Kubo R, lyoda A, et al: Analysis of cell cycle-related proteins in mediastinal lymph nodes of patients with N2-NSCLC obtained by EBUS-TBNA: relevance to chemotherapy response. Thorax 2008, 63:642-647.

34. Karamitopoulou E, Zlobec I, Patsouris E, Peros G, Lugli A: Loss of E-cadherin independently predicts the lymph node status in colorectal cancer. Pathology 2011, 43:133-137.

35. Schwartz B, Melnikova VO, Tellez C, Mourad-Zeidan A, Blehm K, Zhao YJ, et al: Loss of AP-2alpha results in deregulation of E-cadherin and MMP-9 and an increase in tumorigenicity of colon cancer cells in vivo. Oncogene 2007, 26:4049-4058.

36. Arumugam $T$, Ramachandran V, Fournier KF, Wang $H$, Marquis $L$, Abbruzzese $J$, et al: Epithelial to mesenchymal transition contributes to drug resistance in pancreatic cancer. Cancer Res 2009, 69:5820-5828.

\section{Pre-publication history}

The pre-publication history for this paper can be accessed here: http://www.biomedcentral.com/1471-2407/11/135/prepub

doi:10.1186/1471-2407-11-135

Cite this article as: Nordentoft et al:: Increased expression of transcription factor TFAP $2 \alpha$ correlates with chemosensitivity in advanced bladder cancer. BMC Cancer 2011 11:135. 